\title{
LOCAL ADHESIVE SURFACE PROPERTIES STUDIED BY FORCE MICROSCOPY
}

\author{
M. LekKa, J. LekKI, M. Marszalek, Z. Stachura \\ Institute of Nuclear Physics, Radzikowskiego 152, 32-341 Cracow, Poland \\ AND B. CLEFF \\ Institute of Nuclear Physics, Wilhelm-Klemm-Str. 9, 48-149 Münster, Germany \\ Scanning force microscopy was used in the contact mode to determine \\ the adhesion force between a mica surface and a silicon nitride tip. The mea- \\ surements were performed in an aqueous solution of sodium and calcium \\ chlorides. The adhesion force according to the Derjaguin-Landau-Verwey- \\ Overbeek theory depends on the competition between two kinds of forces: \\ van der Waals and electrostatic "double layer". Two different curves of adhe- \\ sion force versus salt concentration were obtained from the experiment with \\ monovalent and divalent ions. The tip-surface adhesion force was deter- \\ mined from a statistical analysis of data obtained from the force vs. distance \\ retracting curves.
}

PACS numbers: $68.35 . G y$

\section{Introduction}

Scanning force microscopy (SFM) offers a possibility of studying the interaction between the scanning tip and investigated surface. In particular, adhesion forces, which are very important in biology because of their role in many vital processes, may be studied. According to the Derjaguin, Landau, Verwey, and Overbeek (DLVO) theory, during the measurements performed in aqueous solution mainly two types of forces appear. These forces are the repulsive electrostatic "double layer" force and the attractive van der Waals one. The competition between them determines the adhesion force.

In this work the force measurements in different salt concentrations of the sodium and calcium chlorides were performed. The salt solutions containing the important in biology cations of $\mathrm{Na}^{+}$and $\mathrm{Ca}^{2+}$ were chosen. As a substrate, the mica surface with its negative charge was used. This assured simplicity of the system and its similarity to the biological surface, which in most cases is also negatively charged. The exact knowledge of the dominant forces investigated here is needed before studying the whole spectrum of the other forces acting in real biological membranes (hydration forces, solvation etc.). 


\section{Material and experimental method}

The SFM used for this experiment was a home-built device working in contact mode [1]. The microscope was equipped with a piezotube scanner. The range of the contracting and the extending of piezotube was $\pm 1.5 \mu \mathrm{m}$. The sample was measured in a liquid cell made of plexiglass. The cantilever deflection caused by the force acting between a tip and a sample was detected using the laser-photodiode system. The laser beam passing through a plexiglass top cover and liquid environment is reflected from cantilever and detected by the photodiode. The loss of the intensity of the laser beam was about $20 \%$ in comparison with measurements performed in air. In this experiment we used standard silicon nitride tips from PSI (Park Scientific Instruments) with spring constants of $0.03 \mathrm{~N} / \mathrm{m}, 0.05 \mathrm{~N} / \mathrm{m}$, and $0.1 \mathrm{~N} / \mathrm{m}$ and tip radius of $20 \mathrm{~nm}$.

The height calibration was performed as follows. First, the measurement of the silicon grating with height steps of $25 \mathrm{~nm}$ was performed. For hard, non-deforming surface (like mica) in the range of our interest (that is up to $10 \mathrm{nN}$ ) the cantilever deflection is the same as the change of the sample position. Therefore the slope of the cantilever deflection curve versus sample position should be 45 degrees. That gives a control over tip-sample distance coordinate. Additionally, the calibration was confirmed by an interferometric experiment using the laser beam reflected from a sloped mirror and interfering with the reflection from the cantilever.

The investigated surface was a cleavage sheet of the muscovite mica. The mica has a negative lattice charge due to the periodic replacement of $\mathrm{Si}$ atoms by Al. In water solution mica surface has a negative charge [2]. The mica sheet was glued onto a cleaned plexiglass holder, magnetically attached to the piezotube. The cleavage of mica was performed in distilled water. Next, the sample was put into the liquid cell for measurements. The environmental liquids were sodium chloride and calcium dichloride dissolved in water. The range of applied concen-

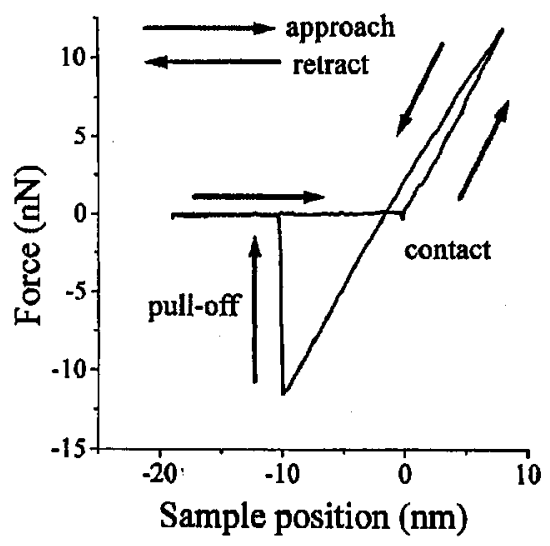

Fig. 1. Principle of adhesion force determination. 
trations varied from $1 \mathrm{mmol}$ to $100 \mathrm{mmol}$. The purified chemicals were bought from POCh S.A. Gliwice. All measurements were done in the room temperature $19-20^{\circ} \mathrm{C}$.

For each experiment at a given salt concentration four sheets of freshly cleaved mica were used and for each sheet several points of measurements were chosen. In every point 25 curves of the cantilever deflection versus position of the sample were collected. Force versus distance plots were obtained from deflection curves using simple formulas: the cantilever deflection (nm) was converted into force by multiplying it by the spring constant and the distance was obtained by subtracting the cantilever deflection from the sample position. Retracting curves were studied in order to calculate the adhesion force, defined as a force needed to separate the tip and the investigated surface (Fig. 1). The mean value of the adhesion force and its variance was obtained from a fit of the Gaussian distribution to the histogram of experimental values of adhesion.

\section{Results and discussion}

Adhesion force is defined as a force needed to separate two bodies from each other. The measurement of "pull-off" force where the tip is detached from the surface may be used for determination of adhesion force value. The attractive van der Waals component of the adhesion can be calculated for mica-water-silicon nitride system assuming the geometry of a sphere coming into contact with a flat plane. For our system, according to the Lifshitz theory [3], the calculated Hamaker constant was $32.3 \times 10^{-20} \mathrm{~J}$. The estimated attractive van der Waals force was about $40 \mathrm{nN}$ for the assumed distance of $D_{0}=0.165 \mathrm{~nm}$ when the Born repulsion occurred. However, the measured pull-off force was much lower than this value. This may be explained by taking into account electrostatic interactions in presence of ions in liquid between the tip and the sample. In this case the adhesion force results as a competition of the attractive van der Waals force and the repulsive electrostatic "double-layer" force. Electrostatic forces may achieve values comparable to the van der Waals interaction and decrease with distance with characteristic Debye length [3]. This effect can reduce the measured "pull-off" (adhesion) force, which is shown in Fig. 2 for measurements performed in different salt concentrations of sodium and calcium chloride solutions. However, it should be noted here that the method used in present work measures the magnitude of attractive force, and in the case of repulsive one the measurement gives a value of zero. Having this in mind, we may conclude from Fig. 2 that for $\mathrm{NaCl}$ solution the adhesion values close to zero were measured, in contrary to the $\mathrm{CaCl}_{2}$ solution, where a clear maximum reaching $3.5 \mathrm{nN}$ was observed at about $50 \mathrm{mmol}$ concentration. The difference between $\mathrm{CaCl}_{2}$ and $\mathrm{NaCl}$ solutions behaviour origins from the different kinds of ions: $\mathrm{Ca}$ ions are divalent, and $\mathrm{Na}$ - monovalent. The corresponding Debye length and repulsive electrostatic force is lower for $\mathrm{Ca}^{++}$ions than for $\mathrm{Na}^{+}$ions so the adhesion force resulting from summation of the van der Waals and electrostatic interactions should be larger for $\mathrm{Ca}^{++}$than for $\mathrm{Na}^{+}$. This is in agreement with $\mathrm{NaCl}$ results, where a close to zero pull-off force was measured. Indeed, a repulsive interaction was observed in our experiments in the force-distance curves for the approaching tip. However, this simple explanation basing on interplay of two main forces is not 

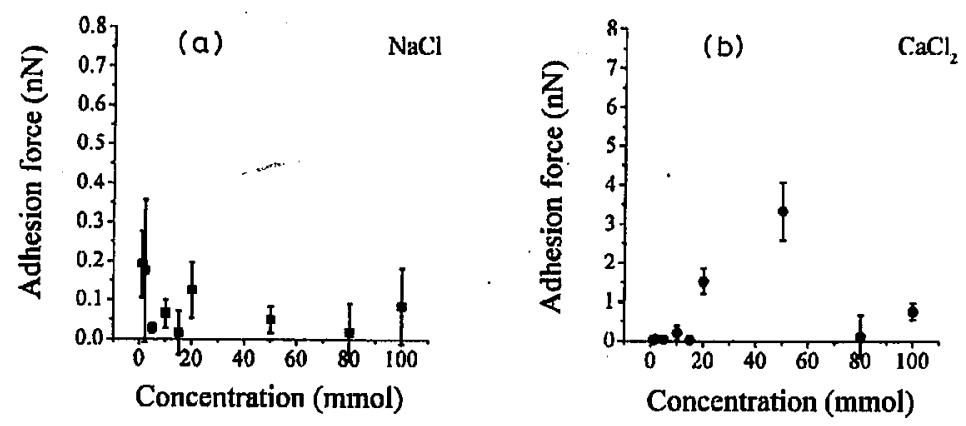

Fig. 2. Dependence between adhesion force and (a) $\mathrm{NaCl}$ and (b) $\mathrm{CaCl}_{2}$ salt concentration.

sufficient to explain a non-monotonic behaviour and a clear appearance of a maximum of adhesion present for the $\mathrm{CaCl}_{2}$ solution curve. At low salt concentrations the repulsive electrostatic term is stronger than the van der Waals component and the resulting adhesion force is close to zero. With increasing salt concentration the repulsion decreases and positive adhesion values are observed. In contrast to the van der Waals interactions, weakly dependent on the electrolyte concentration, the electrostatic force is strongly decreasing with increasing salt concentration. Surprisingly, with further increase in $\mathrm{CaCl}_{2}$ concentration, adhesion force vanishes again. This may be explained by introduction of the hydration forces, observed already in similar systems [4, 2]. Hydration force stems from binding of water by counterions adsorbed at the surfaces. Water molecules must bè removed when the surfaces approach, which results in additional repulsive force with a characteristic length of about one $\mathrm{nm}$. This effect may be particularly significant for divalent ions with stronger bonding to water molecules. Hydration forces were clearly observed especially at high ionic concentrations. However, they are present also at lower salt concentrations and contribute to total interaction, modifying the minimum tip-surface distance during the contact. Therefore, even when not observed during approach they may influence retracting behaviour. Their role increases with salt concentration and they are responsible for the decay of adhesion force in $\mathrm{CaCl}_{2}$, as seen in Fig. 2.

This work was partially supported by a grant No. 2 P03B 03312 of the Committee for Scientific Research (Poland).

\section{Rèferences}

[1] M. Lekka, J. Lekki, A.P. Shoulyarenko, B. Cleff, J. Stachura, Z. Stachura, Polish J. Pathol. 47, 51 (1996).

[2] R.M. Pashley, J. Colloid Interface Sci. 83, 531 (1981).

[3] J. Israelachvili, Intermolecular and Surface Forces, Academic Press, San Diego 1992.

[4] H.J. Butt, Biophys. J. 60, 1438 (1991). 